2007, 111, 6589-6592

Published on Web 04/18/2007

\title{
Photo-Driven Expulsion of Molecules from Mesostructured Silica Nanoparticles
}

\author{
Sarah Angelos, ${ }^{\dagger}$ Eunshil Choi, ${ }^{\dagger}$ Fritz Vögtle, ${ }^{\ddagger}$ Luisa De Cola, ${ }^{\S}$ and Jeffrey I. Zink ${ }^{*, \dagger}$ \\ Department of Chemistry and Biochemistry, University of California Los Angeles, Los Angeles, California \\ 90095, Kekulé-Institut für Organische Chemie und Biochemie der Universität Bonn, Gerhard-Domagk-Strasse \\ 1, D-53121 Bonn, Germany, and Westfälische Wilhelms-Universität Münster, Physikalisches Institut, \\ Mendelstrasse 7, D-48149 Münster, Germany
}

Received: January 27, 2007; In Final Form: March 3, 2007

\begin{abstract}
Azobenzene derivatives act as both impellers and gatekeepers when they are tethered in and on mesoporous silica nanoparticles. Continuous excitation at $457 \mathrm{~nm}$, a wavelength where both the cis and trans conformers absorb, produces constant isomerization reactions and results in continual dynamic wagging of the untethered terminus. The $2 \mathrm{~nm}$ diameter pores are loaded with luminescent probe molecules, azobenzene motion is stimulated by light, and the photoinduced expulsion of the probe from the particles that is caused by the motion is monitored by luminescence spectroscopy. The light-responsive nature of these materials enables them to be externally controlled such that the expulsion of dye molecules from the mesopores can be started and stopped at will. These results open the possibilities of trapping useful molecules such as drugs and releasing them on demand.
\end{abstract}

\section{Introduction}

Control of molecular transport in, through, and out of mesopores has important potential applications in nanoscience including fluidics and drug delivery. Surfactant-templated silica ${ }^{1}$ is a versatile material in which ordered arrays of mesopores can be easily synthesized, providing a convenient platform for attaching molecules that undergo large amplitude motions to control transport. Mesostructured silica is transparent (for photocontrol and spectroscopic monitoring), and can be fabricated into useful morphologies (thin films, ${ }^{2}$ particles ${ }^{1,3}$ ) with designed pore sizes and structures. One successful method of controlling transport uses the photoinduced cis-trans isomerization of $\mathrm{N}=\mathrm{N}$ bonds in azobenzene derivatives tethered to the interiors of mesopores. To date, understanding of the lightresponsive behavior of azobenzene-modified materials has been based on a static mechanism, where the effective pore sizes are varied by azobenzene existing in the trans or cis conformation. In this letter, it is shown that continuous excitation at $457 \mathrm{~nm}$, a wavelength where both the cis and trans conformers absorb, produces constant isomerization reactions that cause continual dynamic wagging of the untethered terminus and impel molecules through the pores. In addition, it is shown that the dynamic control of transport can be made to occur in $400 \mathrm{~nm}$ diameter particles containing $\sim 2 \mathrm{~nm}$ diameter pores.

Mesostructured inorganic materials functionalized with azobenzene $\mathrm{e}^{4-9}$ have received significant attention owing to the photoactive responses of these hybrids, including control of the d-spacing of mesostructured materials. ${ }^{5}$ Zeolitic membranes

* To whom correspondence should be addressed. E-mail: zink@ chem.ucla.edu.

$\dagger$ University of California Los Angeles.

$\doteqdot$ Kekulé-Institut für Organische Chemie und Biochemie der Universität Bonn.

$\S$ Westfälische Wilhelms-Universität Münster, Physikalisches Institut. containing azobenzene were reported to exhibit photoswitchable gas permeation properties resulting from the trans-cis isomerization of azobenzene. ${ }^{9}$ Mesostructured silicates synthesized with azobenzene-bridged pores exhibit light-responsive changes in adsorption ability correlating with the dimensional changes of azobenzene that occur upon photoisomerization. ${ }^{7}$ Additionally, the transport rate of ferrocene derivatives through an azobenzene-modified cubic-structured silica film to an electrode was photoresponsively controlled by changing the effective pore size. ${ }^{6}$

A different type of transport control utilizes molecular machines with moving parts based on large amplitude molecular motions that are attached to the pore openings and act as gatekeepers. Gatekeepers based on supramolecular motifs where a cyclic component moves relative to a stationary thread, ${ }^{10-13}$ on intermolecular dimerization of coumarin, ${ }^{14,15}$ and on forming and breaking disulfide bonds ${ }^{16}$ have been demonstrated. Sources of energy that have been used include $\mathrm{pH}$ changes, ${ }^{11}$ redox chemical energy, ${ }^{12}$ and competitive binding. ${ }^{13}$

Herein, we demonstrate that the dynamic motion of azobenzene derivatives can be used to control the transport of molecules trapped in the mesopores of silica nanoparticles. We report the use of azobenzene derivatives as both impellers and gatekeepers in and on mesoporous silica nanoparticles, such that guest molecules are expelled from the particles under photocontrol. We designed spherical particles with diameters of about 400 $\mathrm{nm}$, a small azobenzene derivative, AzoH (Figure 1), to attach to the pore interiors, and a larger azobenzene derivatized with a G1 Frechet dendron (AzoG1) to attach to the pore openings. Our prior photophysical studies have shown that switching of immobilized azobenzenes occurs inside of mesopores; the trans to cis isomerization quantum yield at $450 \mathrm{~nm}$ is 0.36 and that for cis to trans is $0.64 .{ }^{17}$ Continuous excitation at this wavelength 
a)

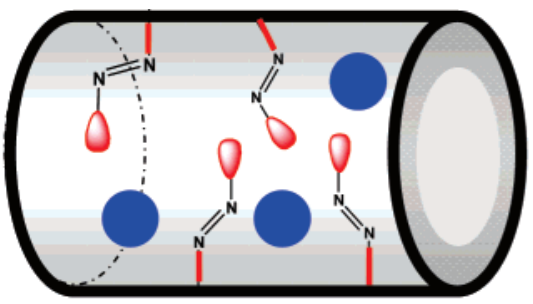<smiles>O[Hg]</smiles>

$\mathrm{AzoH}$<smiles>O=C(O)OCc1cc(OCc2ccccc2)cc(OCc2ccccc2)c1</smiles>

b)

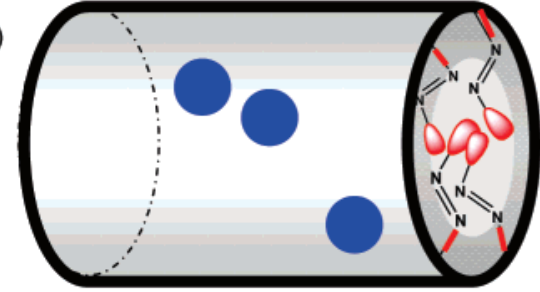<smiles>C#Cc1ccc(NC(=O)N(CC)CCOCC)cc1</smiles><smiles>C#COCCOCC</smiles>

Rhodamine 6G<smiles>CCCN(CCCOCC)C(=O)OCc1ccc(C)cc1</smiles><smiles>O=c1cc(Br)c2cc3c4c(c2o1)CCCN4CCC3</smiles>

Coumarin 540A

Figure 1. Photoresponsive materials functionalized with azobenzene derivatives. (a) Materials prepared by the $\mathrm{CCM}$ are derivatized with AzoH; (b) materials prepared by the PSMM are derivatized with AzoG1. For each system, the moveable phenyl ring of the azobenzene machine is illustrated by the red inverse teardrop, the tethered phenyl ring of the azobenzene machine by the red vertical bar, and the impelled molecule by the blue circle.

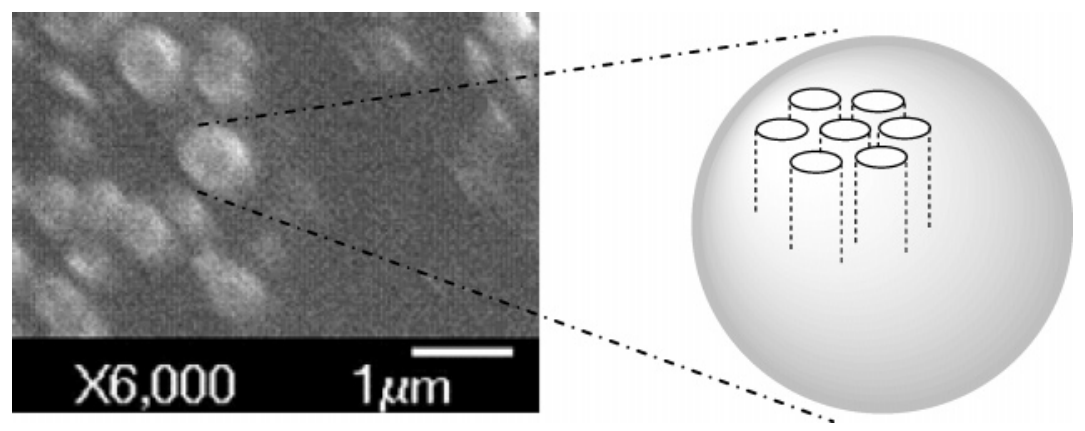

Figure 2. Scanning electron microscopy image of silica nanoparticles and illustration of the $2 \mathrm{D}$ hexagonal mesostructure. (The $\sim 2 \mathrm{~nm}$ diameter pores are not drawn to scale.)

produces constant isomerization reactions and results in continual dynamic wagging of the untethered terminus. In the experiments reported here, azobenzene-modified pores are loaded with luminescent probe molecules, azobenzene motion is stimulated by light, and luminescence spectroscopy is used to monitor the photoinduced expulsion of the probe from the particles that is caused by the azobenzene motion. The relative efficiency of expulsion of the small probe molecules during radiation to retention in the dark is dependent on the position of the azobenzene in the pore, the concentration, and the size of the azobenzene moving part.

\section{Experimental Section}

The solid supports for the azobenzene machines are $\sim 400$ $\mathrm{nm}$ diameter particles that contain ordered two-dimensional (2D) hexagonal arrays of tubular pores ( $4 \mathrm{~nm}$ lattice spacing) prepared by a base catalyzed sol-gel method. ${ }^{1,3}$ The pores are templated by cetyltrimethylammonium bromide (CTAB) surfactants, and tetraethylorthosilicate (TEOS) is used as the silica precursor. Empty pores are obtained by template removal using solvent extraction or calcination. The ordered structure of the mesopores is confirmed by X-ray diffraction (Supporting Information) and the particle morphology by scanning electron microscopy (Figure 2).

Two synthetic approaches were chosen to derivatize the silica in the desired region. To evenly derivatize the interiors of the mesopores, azobenzene was first coupled to the linker molecule isocyanatopropyltriethoxysilane (ICPES), and the machine-linker species was then added to the sol during particle synthesis and allowed to co-condense into the silica framework. The template was removed by solvent extraction. This synthetic approach will be termed the co-condensation method (CCM). To attach the AzoG1 primarily at the pore openings, the calcined mesostructed particles were treated with ICPES followed by coupling. The large azobenzenes cannot penentrate deep inside the pores and the first to react block access to the rest. This approach will be termed the postsynthesis modification method (PSMM). For all the syntheses, reagents were purchased from Aldrich and used as received with the exception of PhMe and ICPES, which were purified by distillation. The synthesis of AzoG1 has been previously reported. ${ }^{17}$

Preparation of AzoH-Modified Materials via the CCM. The synthesis of AzoH-modified materials is derived from a previously reported synthetic methodology. ${ }^{18}$ 4-Phenylazoaniline was first reacted with ICPES to form a carbamide linkage by refluxing $0.2840 \mathrm{~g}$ of the azo with $1.42 \mathrm{~mL}$ of ICPES in $10 \mathrm{~mL}$ of EtOH under $\mathrm{N}_{2}$ for $4 \mathrm{~h}$. During the coupling reaction, a surfactant solution ${ }^{3}$ was prepared in the other flask: $2.0 \mathrm{~g}$ of CTAB, $7.0 \mathrm{~mL}$ of $2 \mathrm{M} \mathrm{NaOH}$, and $480 \mathrm{~g}$ of the deionized $\mathrm{H}_{2} \mathrm{O}$ were mixed and stirred for $30 \mathrm{~min}$ at $80{ }^{\circ} \mathrm{C}$. To this solution, $9.34 \mathrm{~g}$ of the TEOS and the coupled AzoH-ICPES machine were slowly added with vigorous stirring. After $2 \mathrm{~h}$ of stirring 
at $80^{\circ} \mathrm{C}$, the particles were filtered and thoroughly washed with $\mathrm{MeOH}$ and deionized $\mathrm{H}_{2} \mathrm{O}$. Template removal was accomplished by suspending $1 \mathrm{~g}$ of the as-synthesized particles in $100 \mathrm{~mL}$ of $\mathrm{MeOH}$ with $1 \mathrm{~mL}$ of concentrated $\mathrm{HCl}$ and heating at $60{ }^{\circ} \mathrm{C}$ for $6 \mathrm{~h}$.

Preparation of AzoG1-Modified Materials via the PSMM. Pure mesoporous silica nanoparticles were prepared according to published literature procedure. ${ }^{3}$ The CTAB surfactant was removed by calcination at $550{ }^{\circ} \mathrm{C}$ for $5 \mathrm{~h}$. Attachment of the ICPES linker was accomplished by suspending $100 \mathrm{mg}$ of the calcined particles in $10 \mathrm{~mL}$ of a $10 \mathrm{mM}$ solution of ICPES in dry PhMe and refluxing for $12 \mathrm{~h}$ under $\mathrm{N}_{2}$. ICPES-modified particles were filtered and thoroughly washed with $\mathrm{PhMe}$ and then were placed in a $1 \mathrm{mM}$ solution of AzoG1 in PhMe and refluxed for $12 \mathrm{~h}$ under $\mathrm{N}_{2}$. The AzoG1-modified particles were recovered by filtration, washed thoroughly with $\mathrm{PhMe}$, and then dried under vacuum.

\section{Results and Discussion}

To use the azobenzene motion as an impeller, the small AzoH was attached onto the pore interiors using the CCM. Real time measurements of the rate of expulsion of two different dyes, Coumarin 540A and Rhodamine 6G, were made. The pores were loaded with dye molecules by soaking the particles in $1 \mathrm{mM}$ solutions of the dye overnight and then were washed to remove adsorbed molecules from the surface. Fifteen milligrams of dyeloaded particles were placed in the bottom of a cuvette and 12 $\mathrm{mL}$ of $\mathrm{MeOH}$ was carefully added. A $1 \mathrm{~mW}, 457 \mathrm{~nm}$ probe beam directed into the liquid was used to excite dissolved dye molecules that were released from the particles (Supporting Information). The spectrum was recorded as a function of time at $1 \mathrm{~s}$ intervals. After $5 \mathrm{~min}$, a $9 \mathrm{~mW}, 457 \mathrm{~nm}$ excitation beam was used to directly irradiate the functionalized particles and excite the azobenzenes' motion. Plots of the dissolved dye luminescence intensity at the emission maximum as functions of time (the release profiles) indicate that the particles hold the guest molecules but expel them when stimulated (Figure 3a). As a control experiment to verify that azobenzene excitation drives the release, the particles were irradiated with equal power at a wavelength $(647 \mathrm{~nm})$ at which the azobenezene does not absorb. The red light had no effect on the release. These results demonstrate that the system only responds to wavelengths that drive the large amplitude azobenzene motion.

The expulsion of molecules from pores containing azobenzene molecules attached internally probably involves an "impeller" mechanism. Prior to excitation, dye molecules are held inside the particles because the pores are considerably congested by the static azobenzene machines and a facile pathway for escape is not available. Excitation of the azobenzenes causes them to wag back and forth, effectively imparting motion to the trapped dye molecules and allowing them to traverse the pore interior until they escape. The concentration at which azobenzene machines are tethered to the pore interiors determines the amount of congestion inside the mesopores, and therefore affects the ability to trap dye molecules in the dark. The effective concentration of the AzoH machines tethered inside the mesopores can be varied by changing the amount of the AzoHICPES precursor that is added to the TEOS sol during particle synthesis. When particles are prepared such that the concentration of azobenzene molecules doped into the pores is decreased by a factor of 3 , very slow diffusion of the dye molecules through the pores occurs in the dark and the system is leaky. It is likely that the decreased amount of azobenzene creates enough free space inside the mesopore such that the dye molecules can diffuse around in the dark, and are never completely trapped.
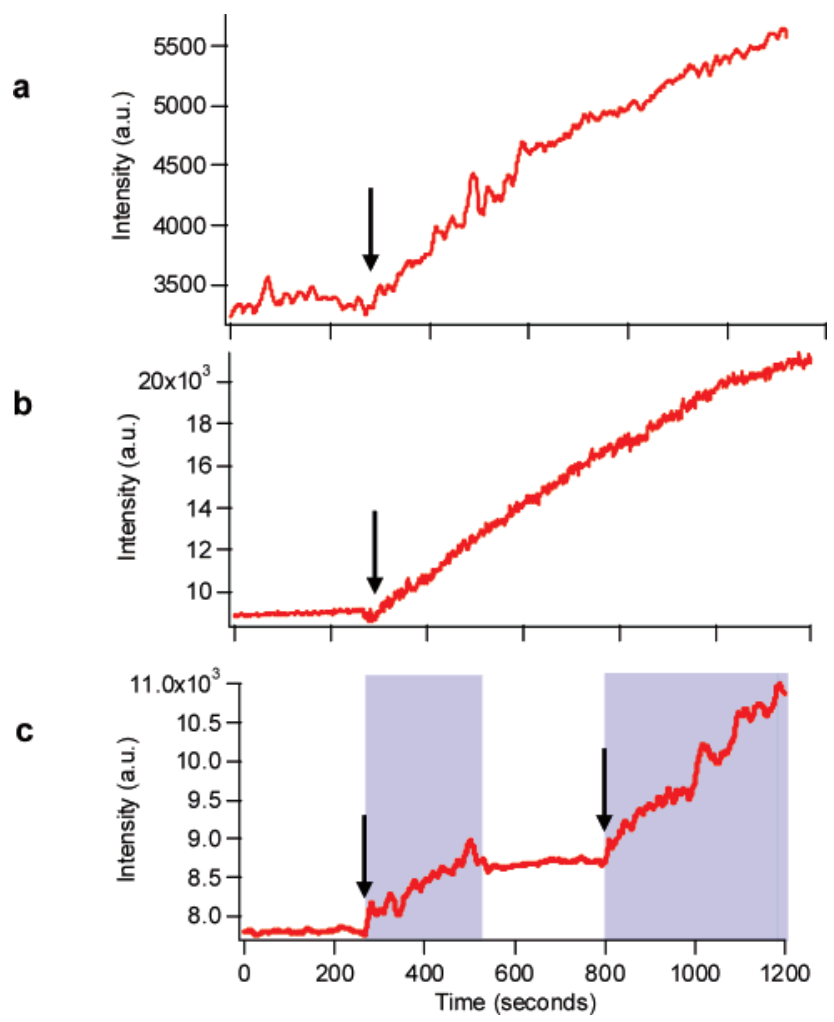

Figure 3. Plots of the luminescence intensity of Coumarin 540A at $540 \mathrm{~nm}$ in solution as a function of time measured at $1 \mathrm{~s}$ intervals. The arrows indicate when the azobenzene excitation light $(457 \mathrm{~nm})$ is turned on. Release profile of Coumarin 540A from (a) AzoH-modified particles prepared by the CCM; $(\mathrm{b}, \mathrm{c})$ AzoG1 modified particles prepared by the PSMM. Profile (c) demonstrates the on-off response to $457 \mathrm{~nm}$ excitation. Shaded regions indicate periods of time at which the azobenzene excitation light is on.

A second method of exploiting dynamic motion is to attach larger azobenzene derivatives at the pore orifices such that the machines can gate the pore openings in the dark. Static large molecules clog the entrances, but dynamic movement could provide intermittent openings for small molecules to slip through. In this gatekeeping approach, the size of the machine selected is a critical factor affecting nanovalve operation. The azobenzene derivative must be sufficiently large such that it can block the nanopore entrances when it is static and mobile enough when irradiated to provide openings through which molecules can escape. AzoG1 was selected because its $1 \mathrm{~nm}$ size suggested that several would be sufficient to block the 2 $\mathrm{nm}$ pores. Minimal leakage of probe molecules is observed prior to excitation but irradiation allows rapid escape (Figure 3b). The smaller derivative AzoH does not sufficiently block the openings and leakage is observed when the molecules are static.

The fact that the dynamic motion responsible for controlling molecular transport can be photoresponsively turned on and off enables the systems to be externally regulated such that the expulsion of dye molecules from the mesopores can be started and stopped at will. The release profile of Coumarin 540A from AzoG1-treated particles where the excitation is sequentially turned on and off is shown in Figure 3c. The pore openings are adequately blocked in the dark and dyes are expelled from the particles only upon excitation of the AzoG1. Remote control of the flow of molecules out of mesopores is thus demonstrated.

\section{Summary}

The functional nanoparticles described here utilize the photocontrollable static and dynamic properties of azobenzene 
derivatives in and on mesopores. Luminescent probe molecules enable the function to be sensitively monitored. These results open up possibilities of trapping useful molecules such as drugs and releasing them on demand.

Acknowledgment. The authors thank the U.S. National Science Foundation CHE 0507929 and DMR 0346601 (J.I.Z.) and EC project IST-2001-35503 (L.D.C. and F.V.) for support of this work.

Supporting Information Available: Synthetic details of AzoH and AzoG1 modified materials. Detailed description of dye-loading process and spectroscopic setup. Characterization of materials including X-ray diffraction, Fourier transform infrared, ${ }^{1} \mathrm{H}$ NMR, light scattering, and UV-vis absorption. Emission spectrum of Coumarin 540A as a function of time. Release profiles of CCM and PSMM materials loaded with Rhodamine $6 \mathrm{G}$. This material is available free of charge via the Internet at http://pubs.acs.org.

\section{References and Notes}

(1) Kresge, C. T.; Leonowicz, M. E.; Roth, W. J.; Vartuli, J. C.; Beck, J. S. Nature 1992, 359, 710-712.

(2) Lu, Y. F.; Ganguli, R.; Drewien, C. A.; Anderson, M. T.; Brinker, C. J.; Gong, W. L.; Guo, Y. X.; Soyez, H.; Dunn, B.; Huang, M. H.; Zink, J. I. Nature 1997, 389, 364-368

(3) Huh, S.; Wiench, J. W.; Yoo, J. C.; Pruski, M.; Lin, V. S. Y. Chem. Mater. 2003, 15, 4247-4256.
(4) Liu, N. G.; Chen, Z.; Dunphy, D. R.; Jiang, Y. B.; Assink, R. A.; Brinker, C. J. Angew. Chem., Int. Ed. 2003, 42, 1731-1734.

(5) Liu, N. G.; Yu, K.; Smarsly, B.; Dunphy, D. R.; Jiang, Y. B.; Brinker, C. J. J. Am. Chem. Soc. 2002, 124, 14540-14541.

(6) Liu, N. G.; Dunphy, D. R.; Atanassov, P.; Bunge, S. D.; Chen, Z.; Lopez, G. P.; Boyle, T. J.; Brinker, C. J. Nano Lett. 2004, 4, 551-554.

(7) Alvaro, M.; Benitez, M.; Das, D.; Garcia, H.; Peris, E. Chem. Mater. 2005, 17, 4958-4964.

(8) Besson, E.; Mehdi, A.; Lerner, D. A.; Reye, C.; Corriu, R. J. P. J. Mater. Chem. 2005, 15, 803-809.

(9) Weh, K.; Noack, M.; Hoffmann, K.; Schroder, K. P.; Caro, J. Microporous Mesoporous Mater. 2002, 54, 15-26.

(10) Hernandez, R.; Tseng, H. R.; Wong, J. W.; Stoddart, J. F.; Zink, J. I. J. Am. Chem. Soc. 2004, 126, 3370-3371.

(11) Nguyen, T. D.; Leung, K. C. F.; Liong, M.; Pentecost, C. D.; Stoddart, J. F.; Zink, J. I. Org. Lett. 2006, 8, 3363-3366.

(12) Nguyen, T. D.; Tseng, H. R.; Celestre, P. C.; Flood, A. H.; Liu, Y.; Stoddart, J. F.; Zink, J. I. Proc. Natl. Acad. Sci. U.S.A. 2005, 102 , 10029-10034.

(13) Leung, K. F. C.; Nguyen, T. D.; Stoddart, J. F.; Zink, J. I. Chem. Mater. 2007, in press.

(14) Mal, N. K.; Fujiwara, M.; Tanaka, Y. Nature 2003, 421, 350353.

(15) Mal, N. K.; Fujiwara, M.; Tanaka, Y.; Taguchi, T.; Matsukata, M. Chem. Mater. 2003, 15, 3385-3394.

(16) Lai, C. Y.; Trewyn, B. G.; Jeftinija, D. M.; Jeftinija, K.; Xu, S.; Jeftinija, S.; Lin, V. S. Y. J. Am. Chem. Soc. 2003, 125, 4451-4459.

(17) Sierocki, P. M. H.; Dragut, P.; Richardt, G.; Vogtle, F.; De Cola, L.; Brouwer, F. A. M.; Zink, J. I. J. Phys. Chem. B 2006, 110, 2439024398.

(18) Liu, N.; Dunphy, D. R.; Rodriguez, M. A.; Singer, S.; Brinker, C. J. Chem. Comm. 2003, 10, 1144-1145. 